\title{
PLANETARY BOUNDARY LAYER HEIGHT VARIABILITY OVER ATHENS, GREECE, BASED ON THE SYNERGY OF RAMAN LIDAR AND RADIOSONDE DATA: APPLICATION OF THE KALMAN FILTER AND OTHER TECHNIQUES (2011-2016)
}

\author{
Dimitrios Alexiou ${ }^{1}$, Panagiotis Kokkalis ${ }^{1}$, Alexandros Papayannis ${ }^{1}$, Francesc Rocadenbosch ${ }^{2,3}$, Athina \\ Argyrouli ${ }^{1}$, Georgios Tsaknakis ${ }^{1}$, and Chris G.Tzanis ${ }^{4}$ \\ ${ }^{1}$ National Technical University of Athens, Laser Remote Sensing Unit, Physics Department, Zografou, Greece, \\ Email: apdlidar@central.ntua.gr \\ ${ }^{2}$ Remote Sensing Laboratory (RSLab), Department of Signal Theory and Communications, Universitat Politècnica \\ de Catalunya, E-08034 Barcelona, Spain \\ ${ }^{3}$ Institute for Space Studies of Catalonia-Aeronautics and Space Research Center, Universitat Politècnica de \\ Catalunya, E-08860 Barcelona, Spain \\ ${ }^{4}$ Division of Environmental Physics and Meteorology, Department of Physics, National and Kapodistrian University \\ of Athens, Athens, Greece
}

\begin{abstract}
In this paper we studied the temporal evolution of the Planetary Boundary Layer height (PBLH) over the basin of Athens, Greece during a 5-year period (20112016) using data from the EOLE Raman lidar system. The lidar data (range-corrected lidar signals-RCS) were selected around 12:00 UTC and 00:00 UTC for a total of 332 cases: 165 days and 167 nights. Extended Kalman filtering techniques were used for the determination of the PBLH. Moreover, several well established techniques for the PBLH estimation based on lidar data were also tested for a total of 35 cases. Comparisons with the PBLH values derived from radiosonde data were also performed. The mean PBLH over Athens was found to be of the order of $1617 \pm 324$ $\mathrm{m}$ at 12:00 UTC and of $892 \pm 130 \mathrm{~m}$ at 00:00 UTC, for the period examined. The mean PBLH growth rate was found to be about $170 \pm 64 \mathrm{~m} \mathrm{~h}^{-1}$ and $90 \pm 17 \mathrm{~m} \mathrm{~h}^{-1}$, during daytime and nighttime, respectively.
\end{abstract}

\section{INTRODUCTION}

The Planetary Boundary Layer (PBL) is the lowest part of the troposphere that is strongly influenced directly by the presence of the Earth's surface and responds to surface forcing with a timescale of about 1 hour or less [1]. The knowledge of the PBLH is very important because air pollutants are trapped within the PBL, thus affecting human health, and atmospheric modeling needs this information to provide air pollution forecasts.

Atmospheric aerosols are present mostly in the lower troposphere where they play a crucial role in the Earth's climate [2]. In the lidar technique aerosols can be used as tracers for the atmospheric motion and for the study of the PBL structure [1].

\section{METHODOLOGY}

Several criteria are currently used to retrieve the PBL height from radiosonde and lidar data, or even from sodar data [3-5]. In this study we will calculate the PBLH (or mixing height) over Athens, Greece, using the extended Kalman filter (KF) technique.

\subsection{THE EXTENDED KALMAN FILTER APPROACH}

The Kalman filter is an adaptive filter inherited from classic control theory [6] that enables the state vector of a dynamic linear system to be estimated and tracked with time (e.g., position coordinates of an aircraft). This filter can also be applied to non-linear systems - as is the case here - via linearization, which gives rise to the Extended Kalman Filter (EKF) technique. The filter operates by minimizing the error between the estimated and the true state vector in a mean-square error sense over time. Because the filter makes use of not only present information (the measurements), but also past estimates, as well as related covariance statistics, it provides an optimal solution over time. Recently, Lange et al., [7], [8], departing from previous works of [9-10], has successfully applied the EKF to estimate the daytime PBLH from tropospheric backscatter lidar and radar signals (returns), respectively. In what follows, we stick to the algorithm and notation given in [7-8]:

In lidar applications, the EKF uses range-corrected backscatter returns, $U(R)$, at successive discrete times, $t_{k}$ (in what follows, the "observables", $\boldsymbol{z}_{\boldsymbol{k}}, k$ a reminder of discrete time; formally, $\boldsymbol{z}_{\boldsymbol{k}}(R), R$ omitted for brevity) as a proxy of the total backscatter coefficient and, in turn, of the atmospheric (aerosol) load. Central to the method is the assumption of an abrupt mixing-layer (ML)-to-free-troposphere (FT) transition in $U(R)$, 
which is modeled by an erf-like function (Fig. 1). The morphology of the erf function of Fig. 1 gives rise to the state vector,

$$
\boldsymbol{x}_{\boldsymbol{k}}=\left[R_{b l, k}, a_{k}, A_{k}, c_{k}\right]^{t} \quad \text { Eq.(1) }
$$

to be estimated at each recursive loop of the filter.

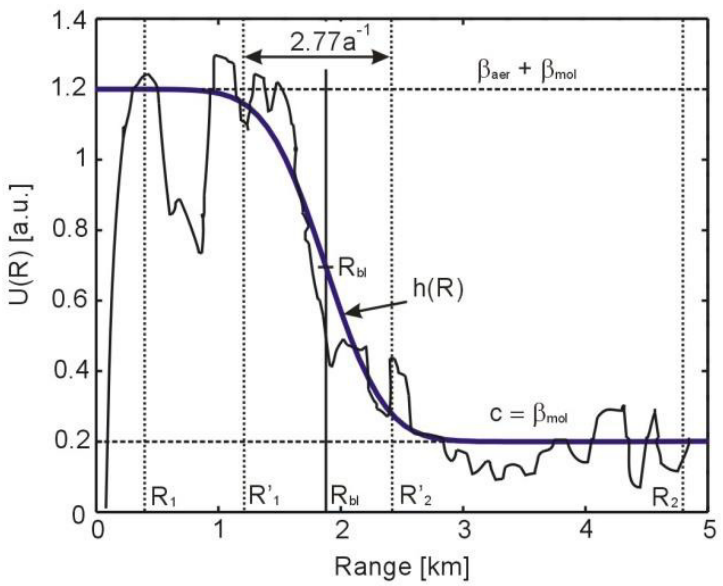

Figure 1. The erf-like transition model. $h(R)$ stands for the total backscatter coefficient, $\mathrm{R}_{\mathrm{bl}}$ is the PBLH, a is the form factor related to the entrainment-zone (EZ) thickness $\left(2.77 \mathrm{a}^{-1}\right), \mathrm{A}$ is the ML-to-FT transition amplitude, and $\mathrm{c}$ is the FT molecular background (adapted from [8]).

The filter requires two models to operate:

(i) The state-vector model, in which the dynamics of the state-vector from time $t_{k}$ to time $t_{k+1}$ are modeled by a Gauss-Markov transition model,

$$
x_{k+1}=x_{k}+w_{k} \quad \text { Eq. (2) }
$$

where $\boldsymbol{w}_{\boldsymbol{k}}$ is the so-called "process noise" or state- noise vector. Because the state vector $\boldsymbol{x}_{\boldsymbol{k}}$ (to be estimated) is a hidden "state" of the atmosphere, additional information is needed from the user's side: (a) an initial guess, $\widehat{x_{0}}=\left[R_{b l, 0}, a_{0}, A_{0}, c_{0}\right]^{t}$, and (b) an estimate of the process-noise covariance matrix, $\boldsymbol{Q}_{\boldsymbol{k}}=E\left[\boldsymbol{w}_{\boldsymbol{k}} \boldsymbol{w}_{\boldsymbol{k}}^{\boldsymbol{t}}\right]$. The latter is approximated in diagonal form, $\boldsymbol{Q}_{\boldsymbol{k}}=$ $\operatorname{diag}\left[\boldsymbol{\sigma}_{Q}^{2}\right], \boldsymbol{\sigma}_{\boldsymbol{Q}}=\left(\sigma_{R b l}, \sigma_{a}, \sigma_{A}, \sigma_{C}\right)$, the latter built from a user-defined intensity factor, $\boldsymbol{\mu}_{\boldsymbol{Q}}$, so that $\boldsymbol{\sigma}_{\boldsymbol{Q}}=\mu_{Q} \widehat{\boldsymbol{x}_{\mathbf{0}}}$. For example, $\sigma_{R b l}$ roughly models the expected standard deviation of the PBLH around its mean value. (c) The filter also requires initialization of the "a priori" error covariance matrix, $\boldsymbol{P}_{\boldsymbol{k}}^{-}=E\left[\boldsymbol{e}_{\boldsymbol{k}}^{-} \boldsymbol{e}_{\boldsymbol{k}}^{-\boldsymbol{t}}\right]$, where $\boldsymbol{e}_{\boldsymbol{k}}^{-}=\boldsymbol{x}_{\boldsymbol{k}}-$ $\widehat{\boldsymbol{x}}_{\boldsymbol{k}}^{-}$is the error vector and $\widehat{\boldsymbol{x}}_{\boldsymbol{k}}^{-}$is the estimated a priori state vector (i.e., prior to assimilating the current measurement at time $\boldsymbol{t}_{\boldsymbol{k}}$ ). Such initialization, $\boldsymbol{P}_{\mathbf{0}}^{-}$, models the expected error on the state-vector initial guess and is provided in the form, $\boldsymbol{P}_{\mathbf{0}}^{-}=\operatorname{diag}\left[\boldsymbol{\sigma}_{\boldsymbol{e}, \boldsymbol{X}}^{\mathbf{2}}\right]$, with $\boldsymbol{\sigma}_{\boldsymbol{e}, \boldsymbol{X}}=$ $\left(\sigma_{e, R b l}, \sigma_{e, a}, \sigma_{e, A}, \sigma_{e, c}\right)$ the user's uncertainty on the state- vector components at $t_{0}$. Likewise, the latter is passed to the filter as an a priori error factor, $\mu_{P}$, so that $\sigma_{e, X}=$ $\mu_{P} \widehat{x_{0}^{-}}$.

(ii) The measurement model, which relates the statevector, $x_{k}$ with the measured observables, $z_{k}$,

$$
\boldsymbol{z}_{\boldsymbol{k}}=\boldsymbol{h}\left(\boldsymbol{x}_{\boldsymbol{k}}\right)+\boldsymbol{v}_{\boldsymbol{k}} \quad \text { Eq. (3) }
$$

where $\boldsymbol{h}\left(\boldsymbol{x}_{\boldsymbol{k}}\right)=\frac{A_{k}}{2}\left\{1-\operatorname{erf}\left[\frac{a_{k}}{\sqrt{2}}\left(R-R_{b l, k}\right)\right]\right\}+c_{k}$

is the erf function of Fig. 1 and $\boldsymbol{v}_{\boldsymbol{k}}$ is the observation noise at time $t_{k}$ with covariance matrix, $\boldsymbol{V}_{\boldsymbol{k}}$ (diagonal). $\boldsymbol{V}_{\boldsymbol{k}}$ is estimated by computing the range-dependent observation noise variance from the signal-to-noise ratio, $\operatorname{SNR}(R)$ [8].

\section{INSTRUMENTATION}

The Laser Remote Sensing Unit (LRSU) of NTUA (EOLE and DIAL lidar systems) is based in Athens $\left(37.96^{\circ} \mathrm{N}, 23.78^{\circ} \mathrm{E}, 220 \mathrm{~m}\right)$, Greece. It is equipped with an advanced 10-wavelength elastic-Raman-DIAL lidar system (Fig. 2) able to perform independent and simultaneous measurements of the vertical profiles of the aerosol backscatter coefficient (at 355, 532 and $1064 \mathrm{~nm}$ ), of the aerosol extinction coefficient (at 355 and $532 \mathrm{~nm}$ ) and of the water vapor and ozone mixing ratio in the troposphere (using the $\mathrm{H}_{2} \mathrm{O}$ Raman channel at $407 \mathrm{~nm}$ and the Differential Absorption Lidar-DIAL technique, respectively) $[11,12]$.

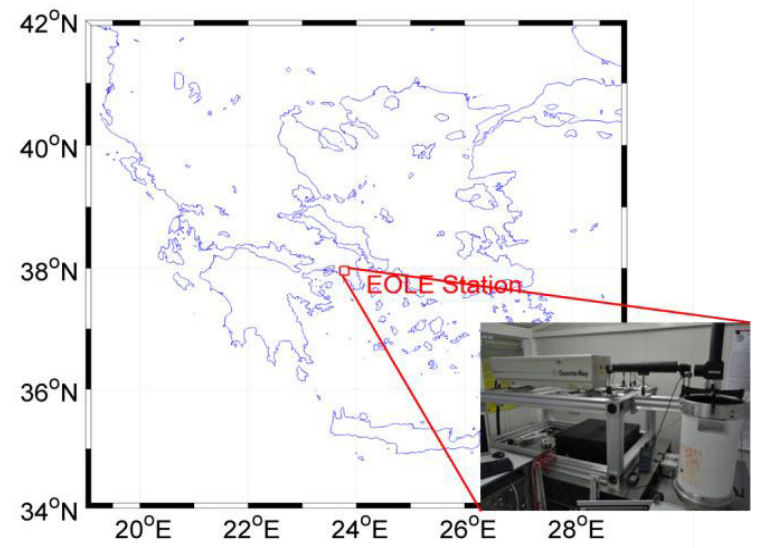

Figure 2. Map of Greece showing the location of the LRSU elastic-Raman lidar (EOLE) system, operating in Athens.

More precisely, the advanced elastic-Raman lidar system (EOLE) of LRSU (Fig. 2) is based on a pulsed $\mathrm{Nd}$ :YAG laser system which emits, simultaneously, high energy pulses at 355-532-1064 nm with $10 \mathrm{~Hz}$ repetition frequency. The laser beam is expanded by a Galilean telescope (x3), before being emitted in the atmosphere. A $300 \mathrm{~mm}$ diameter Cassegrainian telescope collects all elastically backscattered lidar signals, as well as those generated by the spontaneous 
Raman effect (by atmospheric $\mathrm{N}_{2}$ at 387-607 $\mathrm{nm}$ and by $\mathrm{H}_{2} \mathrm{O}$ at $407 \mathrm{~nm}$ ). The lidar signals are then corrected for electronic and atmospheric background noise, prior to range-corrections (RCS) pre-processing.

\section{EXPERIMENTAL RESULTS}

In Fig. 3 we present the monthly variability of the PBLH during daytime (12:00 UTC) and nighttime (00:00 UTC), for the entire 5 years period of measurements (2011-2016), as estimated with the EKF technique from the EOLE lidar signals. During daytime measurements the mean PBLH value is found to be $\sim 1617 \pm 324 \mathrm{~m}$, varying from $982 \mathrm{~m}$ (December) up to $2090 \mathrm{~m}$ (July). During nighttime the PBLH is found to be stable with a mean value of $\sim 892 \pm 130 \mathrm{~m}$,

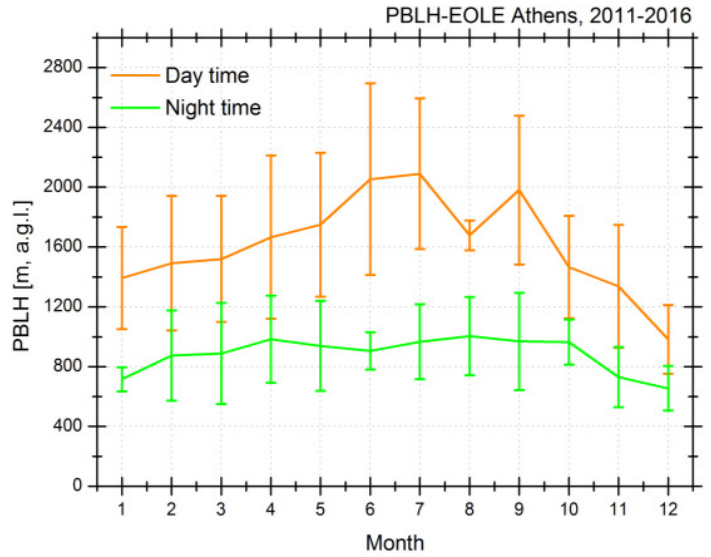

Figure 3. Monthly variability of the PBLH (during daytime and nighttime) as estimated with the EKF technique from EOLE lidar signals (2011-2016).

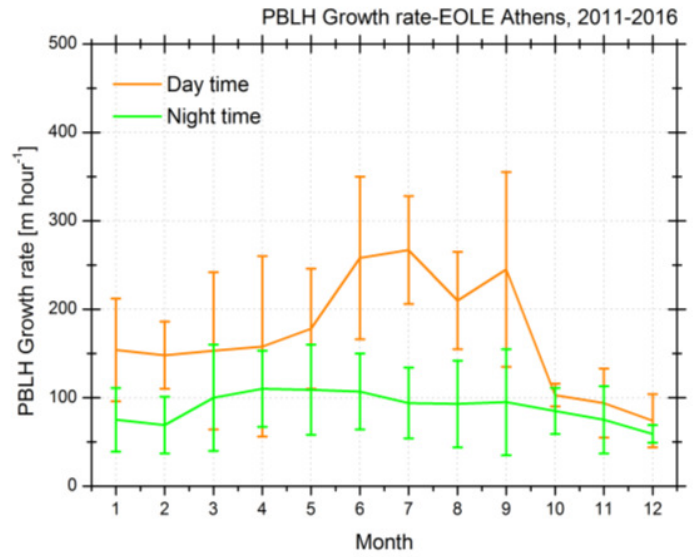

Figure 4. Monthly variability of the PBLH growth rate (during daytime and nighttime) as estimated with the EKF technique from EOLE lidar signals (2011-2016).

The daytime measurements revealed that the growth rate of the PBLH presents a maximum of $\sim 267 \mathrm{~m} \mathrm{~h}^{-1}$ during summer (Fig. 4), where the highest temperature and solar radiance values (measured at $12 \mathrm{~m}$ above ground level, as shown in Fig. 5, orange and blue lines respectively), were recorded for the studied time period. The PBLH along with its growth rate was found to be significantly lower compared to the corresponding values revealed during dust cases. More precisely, the mean growth rate of the PBLH was found to be about $38.8 \mathrm{~m} \mathrm{~h}^{-1}$ lower during cases of dust particles suspended in the atmosphere over Athens, compared to the values presented without dust (Fig. 6).

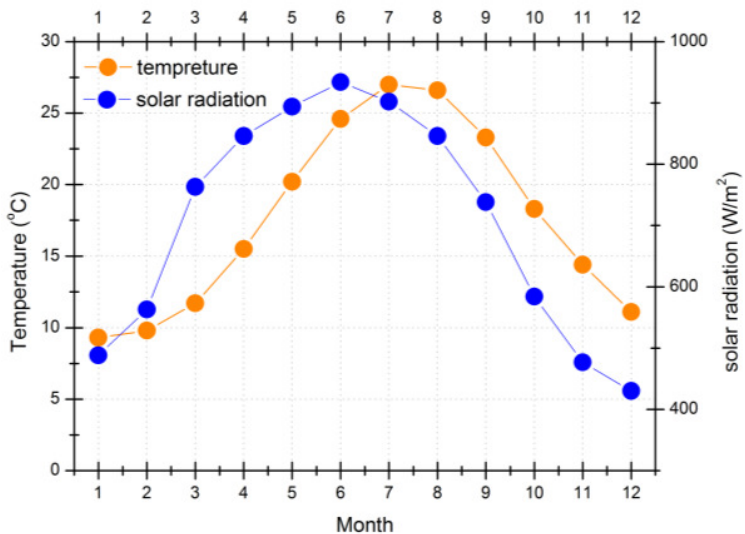

Figure 5. Monthly variability of the air temperature and solar radiation measured at $12 \mathrm{~m}$ above ground level in Athens (2011-2016).

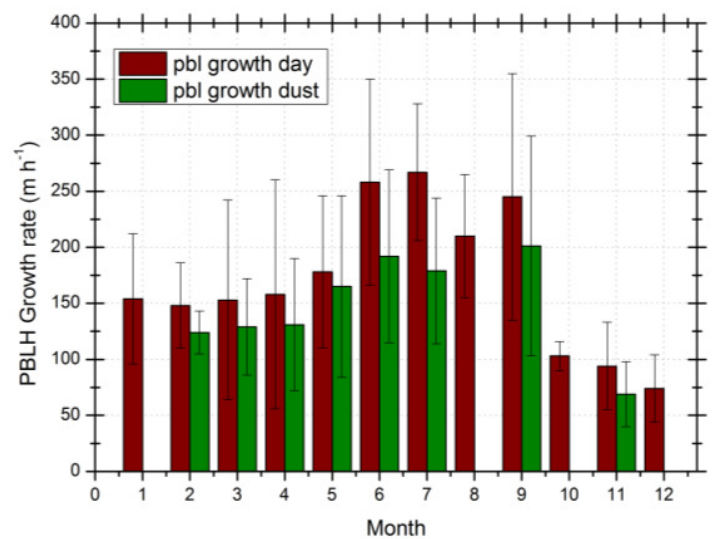

Figure 6. Monthly variability of the PBLH growth rate (during daytime) as estimated with EKF technique from EOLE lidar signals, during dust and non-dust cases over Athens (2011-2016). The error bars are computed from the standard deviation of estimated values within each month.

The values of the PBLH estimated with the EKF technique were further compared to the estimates from other methods using lidar signals (i.e. variance, gradient, inflection, threshold, wavelet covariance methods) and radiosonde data (obtained by the Hellenic National Meteorological Service, HNMS), as shown in Table 1. 
This Table shows that the PBLH derived from the EKF analysis gives the best correlation with the one derived from radiosonde data $\left(\mathrm{R}^{2}=0.87\right.$ for lidar data obtained at 12:00 UTC and $\mathrm{R}^{2}=0.90$ for lidar data averaged within a time interval of $30 \mathrm{~min}$ around 12:00 UTC: 12:00 \pm 0.30 UTC).

Table 1: PBLH comparison between radiosonde and lidar data based on various PBLH retrieving methods (correlation coefficient $\mathrm{R}^{2}$ calculated at 12:00 UTC, without (middle column) and with \pm 30 min temporal averaging (right-hand column)).

\begin{tabular}{|l|c|c|}
\hline & $\begin{array}{c}\mathrm{R}^{2} \\
(12: 00 \\
\mathrm{UTC})\end{array}$ & $\begin{array}{c}\mathrm{R}^{2} \\
(12: 00 \mathrm{UTC}) \\
\pm 30 \mathrm{~min} .\end{array}$ \\
\hline Threshold method & 0.68 & 0.74 \\
\hline Gradient & 0.41 & 0.68 \\
\hline Inflection point & 0.26 & 0.62 \\
\hline Variance & 0.43 & 0.74 \\
\hline Wavelet covariance & 0.79 & 0.84 \\
\hline EKF & 0.87 & 0.90 \\
\hline
\end{tabular}

\section{CONCLUSIONS}

We studied the temporal evolution of the PBLH over Athens, Greece for the period 2011-2016. We found a mean PBLH of $1617 \pm 324 \mathrm{~m}$ (12:00 UTC) and $892 \pm 130$ $\mathrm{m}$ (00:00 UTC). The PBLH growth rate was found to be $\sim 170 \pm 64 \mathrm{~m} \mathrm{~h}^{-1}$ and $90 \pm 17 \mathrm{~m} \mathrm{~h}^{-1}$, during daytime and nighttime, respectively. We also found that the Kalman filter follows much better than the other techniques the PBLH temporal evolution; this is corroborated when compared to the PBLH derived from radiosonde data, where it showed the best correlation $\left(\mathrm{R}^{2}=0.872\right.$ at 12:00 UTC and $\left.\mathrm{R}^{2}=0.901\right)$ for $12: 00 \pm 0.30$ UTC). Thus, we can conclude that the EKF is the most suitable method for PBLH growth studies.

\section{ACKNOWLEDGMENTS}

The research leading to these results has received additional funding from the European Union $7^{\text {th }}$ Framework Program (FP7/2011-2015) and Horizon 2020/2015-2021 Research and Innovation program (ACTRIS) under grant agreement no 262254 and 654109, respectively, as well as from Spanish National Science Foundation and FEDER funds TEC201563832 .

\section{REFERENCES}

[1] Stull, R. B. 1988: An Introduction to Boundary Layer Meteorology, Kluwer Academic Publishers, pp. 666.

[2] IPCC: Climate Change 2013: The Physical Science Basis, Contribution of Working Group
Ito the $5^{\text {th }}$ Assessment Report of the Intergovernmental Panel on Climate Change. Cambridge University Press, Cambridge, United Kingdom and New York, NY, USA, pp. 1535.

[3] Menut, L., et al., 1999: Urban boundary-layer height determination from lidar measurements over the Paris area, Appl. Opt., 38, 945-954.

[4] Seibert, P., et al., 2000: Review and intercomparison of operational methods for the determination of the mixing height, Atmos. Environ., 34, 1001-1027.

[5] Baars, H., et al., 2008: Continuous monitoring of the boundary-layer top with lidar. Atmos. Chem. Phys., 8, 7281-7296.

[6] Kalman, R.E., 1960: A new approach to linear filtering and prediction problems, Trans. American Soc. Mech. Eng. (AMSE) - J. Basic Eng., 82, Series D, pp. 35-45.

[7] Lange, D., et al., 2014: Atmospheric boundary layer height monitoring using a Kalman filter and backscatter lidar returns, IEEE Transactions on Geos. Rem. Sensing, 52, 4717-4728, doi:10.1109/TGRS.2013.2284110.

[8] Lange, D., et al., 2015: Atmospheric-boundarylayer height estimation using a Kalman filter and a frequency-modulated continuous-wave radar, IEEE Trans. Geos. Rem. Sensing, 53, 33383349.

[9] Rocadenbosch, F., et al., 1998: Adaptive filter solution for processing lidar returns: optical parameter estimation, Appl. Opt., 37, pp. 70197034.

[10] Rocadenbosch, F., et al., 1999: Lidar inversion of atmospheric backscatter and extinction-to backscatter ratios by use of a Kalman filter, Appl. Opt., 38, pp. 3175-3189.

[11] Kalabokas, P., et al., 2012: A study on the atmospheric concentrations of primary and secondary air pollutants in the Athens basin performed by DOAS and DIAL measuring techniques, Science Total Environ., 414, pp. 556563.

[12] Kokkalis, P., et al., 2012: The EOLE lidar system of the National Technical University of Athens, Proc. $26^{\text {th }}$ International Laser Radar Conference, pp. 629-632, 25-29 June 2012, Porto Heli, Greece. 